\section{MS5-P36 High-density transfection is superior for production of readily crystallizable glycoproteins in suspension adapted HEK293S GnTI cells: a case study of human lymphocyte receptor LLT1}

Ondřej Vaněk ${ }^{1}$, Jan Bláha ${ }^{1}$, Petr Pachl², Petr Novák ${ }^{1,3}$

1. Department of Biochemistry, Faculty of Science, Charles University in Prague, Hlavova 8, Prague, 12840, Czech Republic

2. Institute of Organic Chemistry and Biochemistry, Academy of Sciences of the Czech Republic, Flemingovo nám. 2, Prague, 16610, Czech Republic

3. Institute of Microbiology, Academy of Sciences of the Czech Republic, Vídeňská 1083, Prague, 14220, Czech Republic

\section{email: ondrej.vanek@natur.cuni.cz}

Human embryonic kidney 293 cell line deficient in $\mathrm{N}$-acetylglucosaminyltransferase I (HEK293S GnTI') is well known tool for recombinant expression of proteins with homogeneous and deglycosylatable $\mathrm{N}$-glycosylation, a feature crucial especially within protein crystallography [1]. So far production protocols using this cell line were based either on lengthy stable cell line generation or transient transfection of adherent cell culture that is costly to scale-up and has reportedly lower expression yields [2].

In this work we have adapted HEK293S GnTI- cell line to growth in suspension and optimized its transient transfection. While transfection at standard cultivation cell density proved very little success we have found out that concentrating the cells to high cell density substantially increases transfection efficiency, greatly enhancing protein yields and creating fast and scalable production process.

We demonstrate this on the production of soluble lectin-like transcript 1 (LLT1, gene clec $2 d$ ) receptor naturally present on natural killer and T-lymphocytes, but upregulated in glioblastoma cells, one of the most lethal tumors, where it acts as a mediator of immune escape. Furthermore, we show that His176Cys mutation is critical for LLT1 stability, leading to reconstruction of disulfide bridge and that LLT1 forms non-covalent homodimer whose dimerization does not depend on presence of its $\mathrm{N}$-glycans [3].

The prepared soluble domain of LLT1 with homogeneous glycosylation was readily crystallized and following optimization of crystal conditions this protein preparation ultimately led to the first structure determination of this receptor described so far [4].

This study was supported by BIOCEV CZ.1.05/1.1.00/02.0109 from the ERDF, by the Czech Science Foundation (project 15-15181S), by the Ministry of Education, Youth and Sports of the Czech Republic (grant LG14009), by Charles University (UNCE 204025/2012, SVV 260079/2014), High Education Development Fund (FRVS 669/2013), BioStruct-X (EC FP7 project 283570) and Instruct, part of the European Strategy Forum on Research Infrastructures (ESFRI) supported by national member subscriptions.

[1] Reeves et al., Proc. Natl. Acad. Sci. USA 99, 2002, 13419-13424. [2] Aricescu et al., Acta Cryst. D62, 2006, 1243-1250. [3] Bláha et al., Protein Expres. Purif. 109, 2015, 7-13. [4] Skálová et al., Acta Cryst. D71, 2015, $578-591$

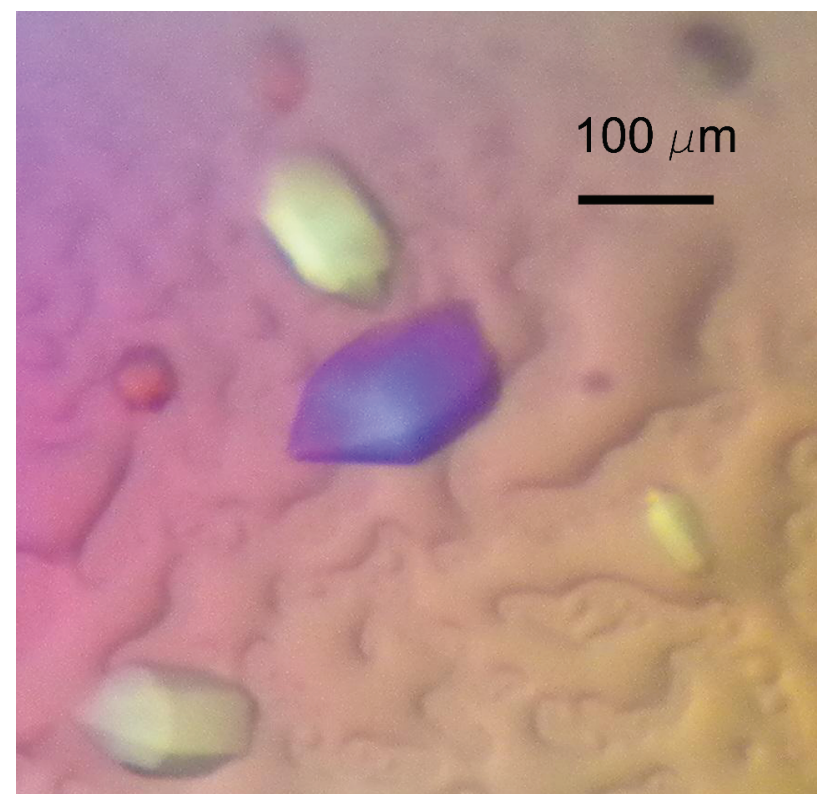

Figure 1. First observed crystals of glycosylated LLT1(H176C) receptor soluble domain produced in HEK293S GnTI- cell line.

Keywords: LLT1; HEK293S GnTI-; C-type lectin-like; NK cell; $\mathrm{N}$-glycosylation; transient transfection; lymphocyte receptor 